\title{
Discovery of the Ternary Nanolaminated Compound Nb2GeC by a Systematic Theoretical-Experimental Approach
}

Per Eklund, Martin Dahlqvist, Olof Tengstrand, Lars Hultman, Jun Lu, Nils Nedfors, Ulf Jansson and Johanna Rosén

\section{Linköping University Post Print}

N.B.: When citing this work, cite the original article.

Original Publication:

Per Eklund, Martin Dahlqvist, Olof Tengstrand, Lars Hultman, Jun Lu, Nils Nedfors, Ulf Jansson and Johanna Rosén, Discovery of the Ternary Nanolaminated Compound $\mathrm{Nb} 2 \mathrm{GeC}$ by a Systematic Theoretical-Experimental Approach, 2012, Physical Review Letters, (109), 3, 035502.

http://dx.doi.org/10.1103/PhysRevLett.109.035502

Copyright: American Physical Society

http://www.aps.org/

Postprint available at: Linköping University Electronic Press

http://urn.kb.se/resolve?urn=urn:nbn:se:liu:diva-79981 


\title{
Discovery of the Ternary Nanolaminated Compound $\mathrm{Nb}_{2} \mathrm{GeC}$ by a Systematic Theoretical-Experimental Approach
}

\author{
Per Eklund, ${ }^{1, *}$ Martin Dahlqvist, ${ }^{1}$ Olof Tengstrand, ${ }^{1}$ Lars Hultman,,${ }^{1}$ Jun Lu, ${ }^{1}$ Nils Nedfors,${ }^{2}$ \\ Ulf Jansson, ${ }^{2}$ and Johanna Rosén ${ }^{1}$ \\ ${ }^{1}$ Department of Physics, Chemistry, and Biology (IFM), Linköping University, IFM, 58183 Linköping, Sweden \\ ${ }^{2}$ Department of Materials Chemistry, The Angström Laboratory, Uppsala University, Box 538, SE-751 21 Uppsala, Sweden
}

(Received 2 April 2012; published 17 July 2012)

\begin{abstract}
Since the advent of theoretical materials science some 60 years ago, there has been a drive to predict and design new materials in silicio. Mathematical optimization procedures to determine phase stability can be generally applicable to complex ternary or higher-order materials systems where the phase diagrams of the binary constituents are sufficiently known. Here, we employ a simplex-optimization procedure to predict new compounds in the ternary $\mathrm{Nb}-\mathrm{Ge}-\mathrm{C}$ system. Our theoretical results show that the hypothetical $\mathrm{Nb}_{2} \mathrm{GeC}$ is stable, and excludes all reasonably conceivable competing hypothetical phases. We verify the existence of the $\mathrm{Nb}_{2} \mathrm{GeC}$ phase by thin film synthesis using magnetron sputtering. This hexagonal nanolaminated phase has $a$ and $c$ lattice parameters of $\sim 3.24 \AA$ and $12.82 \AA$.
\end{abstract}

DOI: 10.1103/PhysRevLett.109.035502

PACS numbers: 61.05.cp, 68.37.Lp, 68.55.Nq

Today's materials science has yielded an unprecedented frequency of new material discoveries. New complex ceramics (borides, carbides, nitrides, and oxides) for a wide range of applications are continuously being synthesized. Much of this work, however, has historically been performed in a trial-and-error manner, and improved theoretical input in guidance of experimental work is essential. In response to this challenge, the last decade has especially seen a tremendous increase in theoretical predictions of hypothetical novel materials. Traditionally, the vast majority of studies calculate only the cohesive energy of the compound itself, which does not give information if the compound is stable relative to any relevant competing phases. This approach yields an unknown local energy minimum in an enormous parameter space, and can very often yield misleading results. A classic example is the prediction of the $\beta-\mathrm{C}_{3} \mathrm{~N}_{4}$ phase with $\mathrm{Si}_{3} \mathrm{~N}_{4}$ structure, which was suggested to be stable and harder than diamond [1]. Extensive experiments were performed and some claimed to have synthesized the $\beta-\mathrm{C}_{3} \mathrm{~N}_{4}$ phase, but it has been presently established that it most likely does not exist [2-4]. A far better approach is to apply exhaustive datamining methods to predict new crystal structures [5-8]. However, their basic premise is that it should be known that a material of a specified chemical composition does exist, followed by determination of its most likely crystal structure. Such approaches to predict new phases thus do not truly reflect on whether hypothetical compounds can be expected to exist experimentally. Consequently, realistic stability calculations versus relevant competing phases are necessary, but when performed they are normally done ad hoc rather than by a systematic approach. The systematic optimization approaches that do exist have mainly been applied to simulate temperature dependence and reaction paths in fully known systems (see, e.g., $[9,10])$.
Here, we apply a linear optimization procedure (based on the simplex method) in which all known competing phases as well as hypothetical competing phases based on neighboring and similar systems are included and the relative stability of any hypothetical compound can be calculated relative to the most stable combination of competing phases $[11,12]$. It should be noted here that the method makes a substantial simplification in accounting only for enthalpy terms, not entropy. Nevertheless, our previous benchmarking confirmed that it gives completely accurate results for existing phases in a fairly large set of well-known carbide and nitride systems [12], but the critical test is whether the method also has predictive power.

As a model system for these general research questions, we have chosen to study $\mathrm{Nb}-\mathrm{Ge}-\mathrm{C}$, where no ternary phases apart from $\mathrm{Nb}_{3} \mathrm{GeC}$ (inverse perovskite) [13] have been reported in the peer-reviewed literature. The binary $\mathrm{Nb}_{5} \mathrm{Ge}_{3}$ can accommodate a substantial amount of carbon and is thus more appropriately described as $\mathrm{Nb}_{5} \mathrm{Ge}_{3} \mathrm{C}_{x}$. In many similar materials systems, there are phases belonging to the class of materials known as $M_{n+1} A X_{n}$ phases ( $n=1-3$, or "MAX phases"), a group of inherently nanolaminated ternary carbides and nitrides $(X)$ of transition metals $(M)$ interleaved with a group 12-16 element (A) [14-19]. Most $M_{n+1} A X_{n}$ phases are $M_{2} A X$ phases (originally called " $H$ phases") and have been known since the 1960s, while the number of $M_{3} A X_{2}$ and $M_{4} A X_{3}$ phases is relatively limited (around a dozen). It is therefore natural to pose the question whether similar phases could exist in the $\mathrm{Nb}-\mathrm{Ge}-\mathrm{C}$ system. This system is also particularly interesting as it would be reasonable to expect superconductivity in a novel $\mathrm{Nb}_{n+1} \mathrm{GeC}_{n}$ phase. Only very few $M_{n+1} A X_{n}$ phases are reported to be superconductors, but those that are mainly tend to be based on the binary superconductor $\mathrm{NbC}$ [15]. Furthermore, these 


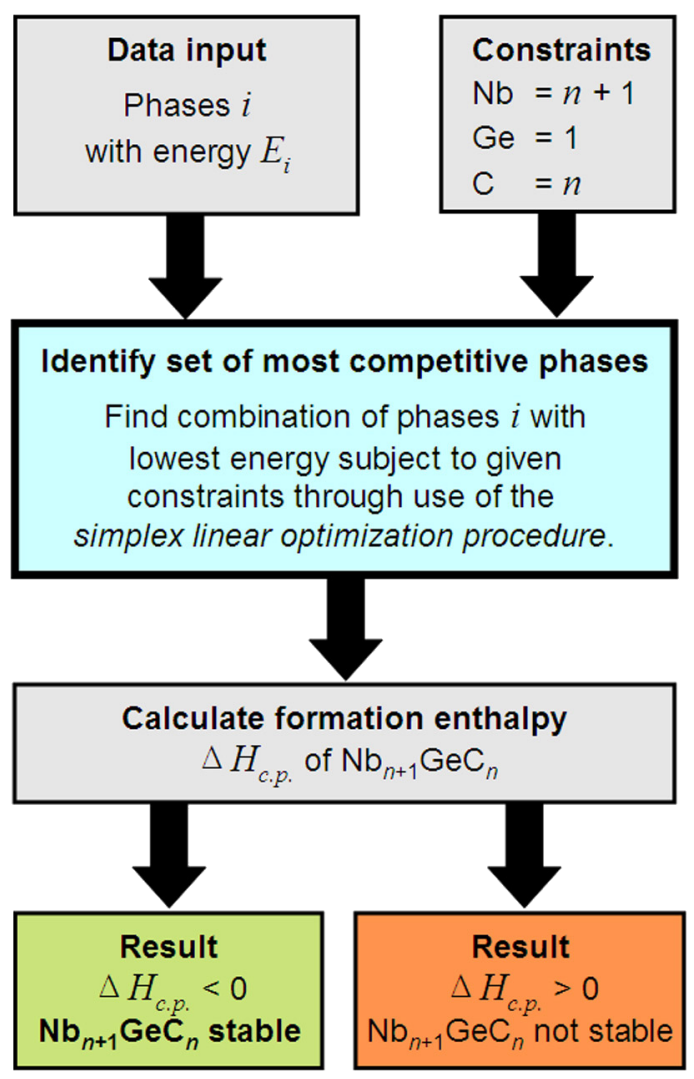

FIG. 1 (color online). Schematic flow chart of the simplex linear optimization procedure used in order to identity the set of most competitive phases with respect to $\mathrm{Nb}_{n+1} \mathrm{GeC}_{n}$.

complex layered phases have enabled the synthesis of new 2D transition-metal "MXene" carbides [20].

To this end, we have performed a systematic investigation of the phase stability of the hypothetical $\mathrm{Nb}_{2} \mathrm{GeC}$, $\mathrm{Nb}_{3} \mathrm{GeC}_{2}$, and $\mathrm{Nb}_{4} \mathrm{GeC}_{3}$. Figure 1 is a flowchart of the optimization procedure. The choice of competing phases is based on known binary phase diagrams $[21,22]$ and the known and hypothetical ternary phases described above. No Ge-C phase is known, and the solubility of $\mathrm{C}$ in $\mathrm{Ge}$ (and vice versa) is negligible. Included phases are schematically shown in Fig. 2. It is a nontrivial task to find the set of phases representing the most competitive ones at a certain composition. We have therefore used the linear optimization procedure introduced in Refs. $[11,12]$, where we confirmed that this method accurately reproduces existing and nonexisting phases in numerous known ternary materials systems. All calculations are based on density-functional theory using the projector augmented wave method [23] as implemented in the Vienna ab initio simulation package (VASP) [24,25]. The Perdew-Burke-Ernzerhof [26] generalized gradient approximation was used for the exchange and correlation functional. Reciprocal-space integration was performed within the Monkhorst-Pack scheme [27] with a plane-wave cutoff energy of $400 \mathrm{eV}$. The $k$-point sampling has been optimized for each phase to obtain a

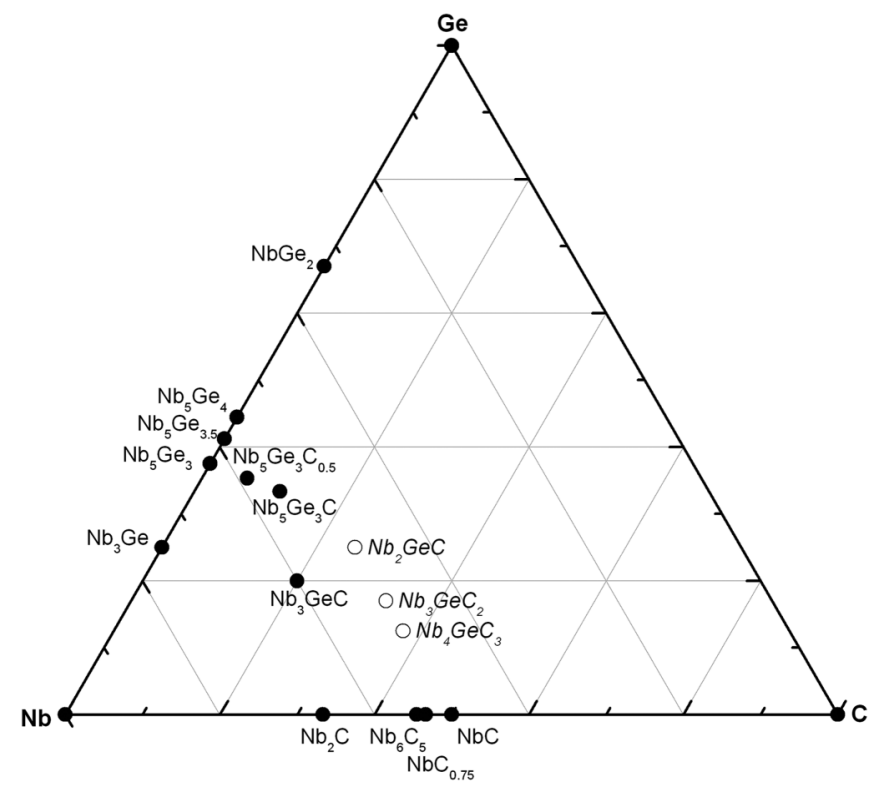

FIG. 2. Schematic phase diagram for the ternary $\mathrm{Nb}-\mathrm{Ge}-\mathrm{C}$ system of known (filled circles) and hypothetical (open circles) phases included in the phase-stability study.

total convergence within $0.1 \mathrm{meV} /$ atom for the total energy. Structural optimizations were performed in terms of unit-cell volumes, $c / a$ ratio (when necessary), and internal atomic positions to minimize the total energy for all phases. Through use of this systematic scheme, we search for the most competitive combination of competing phases at a given elemental composition.

Figure 2 is a schematic phase diagram for the ternary $\mathrm{Nb}-\mathrm{Ge}-\mathrm{C}$ system of known (filled circles) and hypothetical (open circles) phases included in the phase stability study. A full list of all $\sim 20$ included competing phases with structural information is provided as Supplemental Material [28]. The results from total-energy calculations of all competing phases are presented in Table I, including optimized structural parameters. Together with the simplex linear optimization procedure we only find $\mathrm{Nb}_{2} \mathrm{GeC}$ to be stable $\left(\Delta H_{\mathrm{CP}}\right.$ of $-0.018 \mathrm{eV} /$ atom $)$ with $\mathrm{NbGe}_{2}, \mathrm{Nb}_{6} \mathrm{C}_{5}$, and $\mathrm{Nb}_{5} \mathrm{Ge}_{3} \mathrm{C}$ as the set of most competitive phases. Our results also show that the hypothetical $\mathrm{Nb}_{3} \mathrm{GeC}_{2}$ and $\mathrm{Nb}_{4} \mathrm{GeC}_{3}$ are not stable. The calculated cell parameters and unit-cell volume of $\mathrm{Nb}_{2} \mathrm{GeC}$ are $a=3.265 \AA$, $c=12.655 \AA$, and $V=116.83 \AA^{3}\left(58.42 \AA^{3} /\right.$ formula unit), respectively.

TABLE I. Calculated formation enthalpy $\Delta H_{\mathrm{CP}}$ in $\mathrm{eV} /$ atom for $\mathrm{Nb}_{n+1} \mathrm{GeC}_{n}$ phases compared to its identified most competing phases $(\mathrm{CP})$.

\begin{tabular}{ccc}
\hline \hline$n$ & $\Delta H_{\mathrm{CP}}(\mathrm{eV} /$ atom $)$ & Competing phases $(\mathrm{CP})$ \\
\hline 1 & -0.018 & $\mathrm{NbGe}_{2}, \mathrm{Nb}_{6} \mathrm{C}_{5}, \mathrm{Nb}_{5} \mathrm{Ge}_{3} \mathrm{C}_{x}(x=1)$ \\
2 & 0.026 & $\mathrm{Nb}_{2} \mathrm{GeC}, \mathrm{Nb}_{6} \mathrm{C}_{5}, \mathrm{C}$ \\
3 & 0.014 & $\mathrm{Nb}_{2} \mathrm{GeC}, \mathrm{Nb}_{6} \mathrm{C}_{5}, \mathrm{C}$ \\
\hline \hline
\end{tabular}


To test this prediction, we synthesized $\mathrm{Nb}-\mathrm{Ge}-\mathrm{C}$ thin films by dc magnetron sputtering in ultrahigh vacuum (base pressure $\sim 1 \times 10^{-9}$ mbar) onto $\mathrm{Al}_{2} \mathrm{O}_{3}$ (0001) substrates with an $\mathrm{NbC}_{x}$ (111) layer at a substrate temperature of $\sim 800^{\circ} \mathrm{C}$ from elemental targets of $\mathrm{Nb}, \mathrm{Ge}$, and $\mathrm{C}$ in an argon discharge at a pressure of $\sim 0.5 \mathrm{~Pa}$. For details on the synthesis process and chamber, the reader is referred to Refs. [29,30]. The applied power on the sputtering targets was calibrated from known deposition rates and sputtering yields to result in a $\mathrm{Nb}: \mathrm{Ge}: \mathrm{C}$ composition of $2: 1: 1$. Figure 3 is an x-ray diffraction (XRD) $\theta-2 \theta$ scan showing the $\mathrm{NbC}_{x}$ (111) peak and a set of peaks at $13.83^{\circ}, 27.87^{\circ}$, and $42.27^{\circ}$. These peaks are consistent with the positions of the basal-plane $0002,0004,0006$ peaks of the $\mathrm{Nb}_{2} \mathrm{GeC}$ phase, corresponding to a $c$ axis of $12.82 \AA$. XRD pole figures were performed at the positions of the 0002, 0004, and $10 \overline{1} 3$ $\left(13.83^{\circ}, 27.87^{\circ}\right.$, and $\left.38.4^{\circ} 2 \theta\right)$ peaks of this structure and are shown as insets in Fig. 3. The pole figure of the 0004 peak (left inset in Fig. 3) shows diffraction only at the center (i.e., tilt-angle $\chi=0$ ) consistent with basal-plane orientation. The 0002 pole figure was essentially identical to the 0004 pole figure, as expected for basal-plane peaks. The $10 \overline{1} 3$ pole figure (right inset in Fig. 3) shows a set of diffraction peaks at $\chi=57^{\circ}$, corresponding to the angle between $(000 \ell)$ and (1013) planes in the $M_{2} A X$ structure. The low-intensity diffraction feature in the center of this pole figure is due to a minute amount of (1013)-oriented grains (barely detectable in the $\theta-2 \theta$ scans, cf., similar growth results for $\mathrm{Cr}_{2} \mathrm{GeC}$ [31]). Further confirmation of the structure identification was obtained by performing a pole figure of the $10 \overline{1} 6$ peak $\left(53.9^{\circ}\right)$, which yielded a set of diffraction peaks at $\chi=37^{\circ}$, the angle between $(000 \ell)$ and (1016) planes.

Figure 4 shows a high resolution TEM image of the $\mathrm{Nb}_{2} \mathrm{GeC}$ film with the beam aligned along the [112̄0]

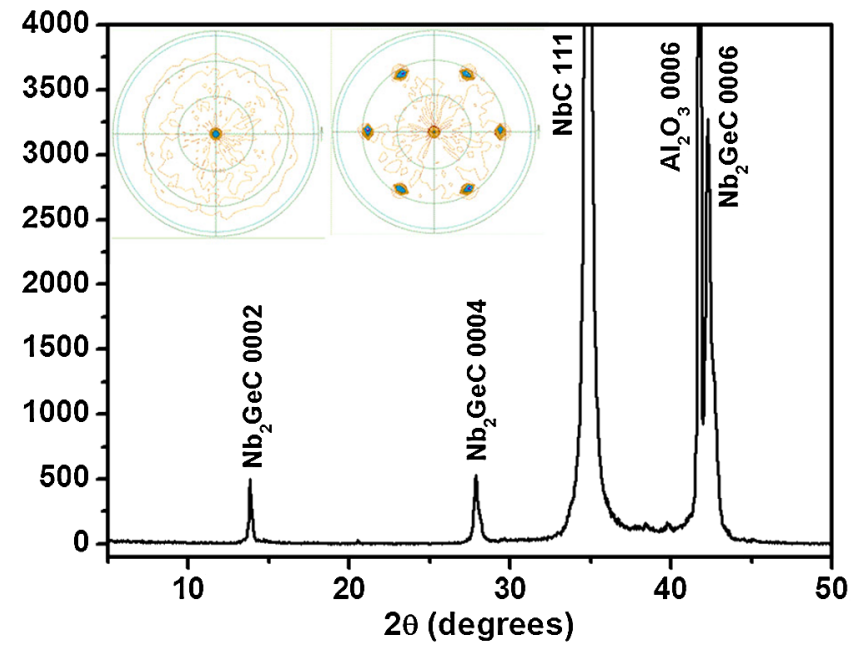

FIG. 3 (color online). XRD $\theta-2 \theta$ scan of $\mathrm{Nb}-\mathrm{Ge}-\mathrm{C}$ thin film on an $\mathrm{Al} 2 \mathrm{O} 3(0001)$ substrate. Insets are pole figures of the 0004 (left) and $10 \overline{1} 3$ (right) peaks of $\mathrm{Nb}_{2} \mathrm{GeC}$. zone axis, unambiguously showing the layered characteristic zigzag structure of the $M_{2} A X$ crystal structure (illustrated in the left side of Fig. 4). These XRD and TEM results prove that the grown phase is indeed $\mathrm{Nb}_{2} \mathrm{GeC}$ with $M_{2} A X$ structure with a $c$ axis of $12.82 \AA$. The $a$ lattice parameter is estimated to be $\sim 3.24 \AA$. The experimentally determined unit-cell volume of $116.45 \AA^{3}$ (58.23 $\AA^{3}$ /formula unit) is very close to the predicted value of $116.83 \AA^{3}$ (58.42 $\AA^{3}$ /formula unit).

Further experiments changing the $\mathrm{Ge}$ and $\mathrm{C}$ content to the compositions closer to $\mathrm{Nb}: \mathrm{Ge}: \mathrm{C}=3: 1: 2$ or $4: 1: 3 \mathrm{did}$ not result in any $\mathrm{Nb}_{3} \mathrm{GeC}_{2}$ or $\mathrm{Nb}_{4} \mathrm{GeC}_{3}$ phases, but rather $\mathrm{Nb}_{2} \mathrm{GeC}$ and $\mathrm{NbC}_{x}$, as predicted. We can therefore conclude that these higher-order phases do not exist, or at least will be very difficult to synthesize, since they are not stable relative to their competing phases. Nevertheless, all three $\mathrm{Nb}_{n+1} \mathrm{GeC}_{n}$ phases are dynamically stable, i.e., stable relative to lattice vibrations as evidenced by the fact that no imaginary phonon frequencies exist in the phonon spectrum (see Supplemental Material [28]). This further underscores the importance of realistic phase-stability calculations in any prediction-based approach.

In conclusion, we have demonstrated the existence of $\mathrm{Nb}_{2} \mathrm{GeC}$ by a combined systematic theoretical optimization procedure and a short set of well-defined experiments. Our work further explains why related hypothetical ternary phases (e.g., $\mathrm{Nb}_{3} \mathrm{GeC}_{2}$ and $\mathrm{Nb}_{4} \mathrm{GeC}_{3}$ ) should not exist, or

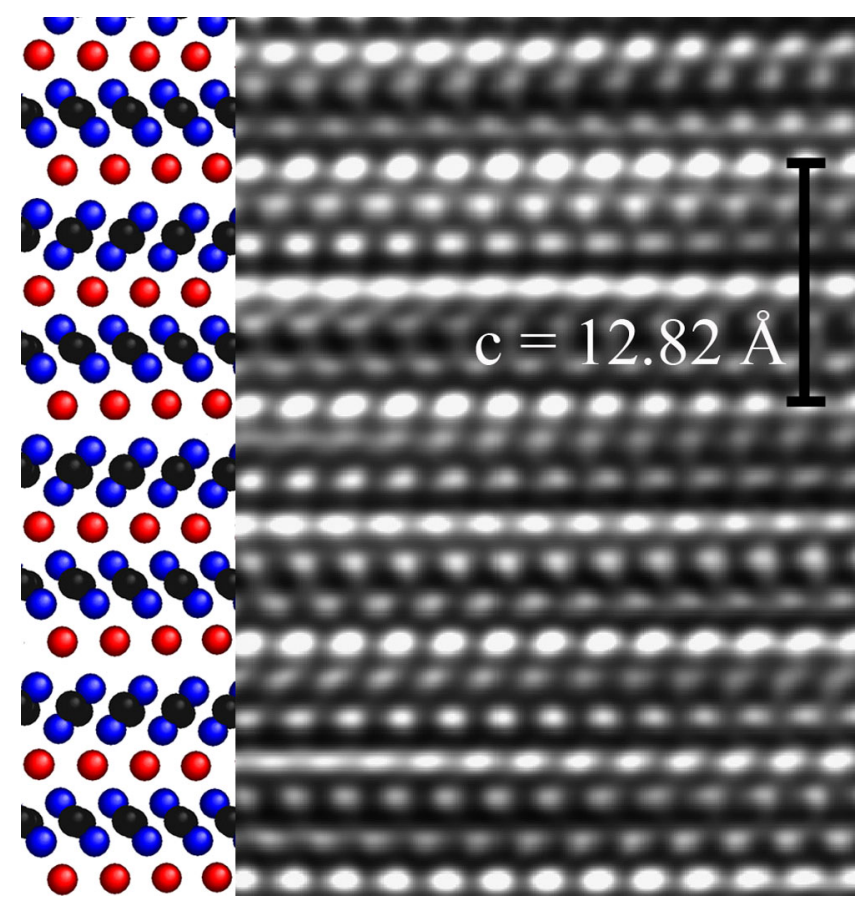

FIG. 4 (color online). High resolution TEM image of the $\mathrm{Nb}_{2} \mathrm{GeC}$ film with the beam aligned along the [1120] zone axis, showing the layered characteristic zigzag structure of the $M_{2} A X$ crystal structure (illustrated to the left of the image). Blue $($ dark gray $)=\mathrm{Nb}$, black $=\mathrm{C}$, red (medium gray $)=\mathrm{Ge}$. 
at least why they should be very difficult to synthesize. The theoretical method used here is in principle generally applicable to complex ternary or higher-order materials systems where at least the phase diagrams of the binary constituents are sufficiently known. Furthermore, it could be combined with a data-mining approach to also allow for the prediction of unknown crystal structures in combination with realistic phase-stability calculations.

The research leading to these results has received funding from the European Research Council under the European Community's Seventh Framework Programme (FP7/2007-2013)/ERC Grant agreement No. [258509], the Swedish Research Council (V. R.), the Swedish Foundation for Strategic Research, and the Swedish Agency for Innovation Systems (VINNOVA) Excellence Center FunMat. The calculations were carried out using supercomputer resources provided by the Swedish National Infrastructure for Computing (SNIC) at the National Supercomputer Center (NSC) and the High Performance Computing Center North (HPC2N). P.E. and M. D. contributed equally to this work.

*Corresponding author. perek@ifm.liu.se

[1] A. Y. Liu and M. L. Cohen, Science 245, 841 (1989).

[2] J. Neidhardt and L. Hultman, J. Vac. Sci. Technol. A 25, 633 (2007).

[3] D. M. Teter and R. J. Hemley, Science 271, 53 (1996).

[4] R. B. Kaner, J. J. Gilman, and S. H. Tolbert, Science 308, 1268 (2005).

[5] S. M. Woodley and R. Catlow, Nature Mater. 7, 937 (2008).

[6] G. Ceder, D. Morgan, C. Fischer, K. Tibbetts, and S. Curtarolo, MRS Bull. 31, 981 (2006).

[7] B. Winkler, C. J. Pickard, V. Milman, and G. Thimm, Chem. Phys. Lett. 337, 36 (2001).

[8] C. Fischer, K. Tibbetts, D. Morgan, and G. Ceder, Nature Mater. 5, 641 (2006).

[9] S. Ong, L. Wang, B. Kang, and G. Ceder, Chem. Mater. 20, 1798 (2008)

[10] R. Akbarzadeh, V. Ozolins, and C. Wolverton, Adv. Mater. 19, 3233 (2007).
[11] M. Dahlqvist, B. Alling, I. A. Abrikosov, and J. Rosén, Phys. Rev. B 81, 024111 (2010).

[12] M. Dahlqvist, B. Alling, and J. Rosén, Phys. Rev. B 81, 220102 (2010).

[13] K. E. Spear and D.F. Palino, Mater. Res. Bull. 18, 549 (1983).

[14] M. W. Barsoum, Prog. Solid State Chem. 28, 201 (2000).

[15] P. Eklund, M. Beckers, U. Jansson, H. Högberg, and L. Hultman, Thin Solid Films 518, 1851 (2010).

[16] J. Y. Wang and Y. C. Zhou, Annu. Rev. Mater. Res. 39, 415 (2009).

[17] M. W. Barsoum and M. Radovic, Annu. Rev. Mater. Res. 41, 195 (2011).

[18] H.-I. Yoo, M.W. Barsoum, and T. El-Raghy, Nature (London) 407, 581 (2000).

[19] M. W. Barsoum, T. Zhen, S. R. Kalidindi, M. Radovic, and A. Murugaiah, Nature Mater. 2, 107 (2003).

[20] M. Naguib, M. Kurtoglu, V. Presser, J. Lu, J. Niu, M. Heon, L. Hultman, Y. Gogotsi, and M. W. Barsoum, Adv. Mater. 23, 4248 (2011).

[21] T. B. Massalski, in Binary Alloy Phase Diagrams (ASM International, Metals Park, Ohio, 1986), Vols. 1 and 2.

[22] H. Okamoto, Phase Diagrams for Binary Alloys (ASM International, Materials Park, Ohio, 2000).

[23] P. E. Blöchl, Phys. Rev. B 50, 17953 (1994).

[24] G. Kresse and J. Hafner, Phys. Rev. B 48, 13115 (1993).

[25] G. Kresse and J. Hafner, Phys. Rev. B 49, 14251 (1994).

[26] J. P. Perdew, K. Burke, and M. Ernzerhof, Phys. Rev. Lett. 77, 3865 (1996).

[27] H. J. Monkhorst and J. D. Pack, Phys. Rev. B 13, 5188 (1976).

[28] See Supplemental Material at http://link.aps.org/ supplemental/10.1103/PhysRevLett.109.035502 for a full list of all 20 included competing phases with structural information, and phonon calculations.

[29] J. Emmerlich, H. Högberg, S. Sasvári, P. O. Å. Persson, L. Hultman, J.-P. Palmquist, U. Jansson, J. M. MolinaAldareguia, and Z. Czigány, J. Appl. Phys. 96, 4817 (2004).

[30] J. Frodelius, P. Eklund, M. Beckers, P. O. A. Persson, H. Högberg, and L. Hultman, Thin Solid Films 518, 1621 (2010).

[31] P. Eklund, M. Bugnet, V. Mauchamp, S. Dubois, C. Tromas, J. Jensen, L. Piraux, L. Gence, M. Jaouen, T. Cabioc'h, Phys. Rev. B 84, 075424 (2011). 